\title{
The diagnostic value of miRNA-141 in prostate cancer: a systematic review and meta-analysis
}

\author{
Yun Ye ${ }^{1}$, Xiaohua Yuan ${ }^{1}$, Jianjun Wang ${ }^{2}$, Yuchen Wang ${ }^{1}$, Suliang Li $^{{ }^{*}}$ \\ ${ }^{1}$ Department of Laboratory Medicine, the First Affiliated Hospital of X''an Medical University, Xi'an, Shaanxi, 710077, China; \\ ${ }^{2}$ Emergency Department, the First Affiliated Hospital of Xi'an Medical University, Xi'an, Shaanxi, 710077, China.
}

\begin{abstract}
MiR-141 has gradually demonstrated its value in the diagnosis of prostate cancer. However, the diagnostic parameters applied in previous studies were different. This systematic review was conducted to explore the diagnostic value of miR-141 in prostate cancer. A comprehensive search of related literature in PubMed, Medline, the Cochrane Library and Embase databases was performed. Seven studies were included which assessed the diagnostic value of miR-141 in patients with prostate cancer up to October 31, 2019. Meta-disc version 1.4 and STATA software version 12.0 were used to analyze the data. The pooled sensitivity and specificity were $0.70(95 \%$ CI 0.64 0.75) and 0.73 (95\% CI 0.64 0.80), respectively. The positive likelihood ratio (PLR) was 2.88 (95\% CI 1.40 5.93), and negative likelihood ratio (NLR) was 0.38 (95\% CI $0.20 \sim 0.71$ ). The pooled diagnostic odds ratio (DOR) of miR-141 for prostate cancer was observed to be 9.94 (95\% CI: 2.55 38.80). The summary area under the receiver operating characteristic (ROC) curve was 0.83 (95\% CI: 0.79 0.86). The results of meta-regression suggested that heterogeneity was mainly derived from patient age. The Fagan nomogram results showed a significant increase when correlating miR-141 with the diagnosis of prostate cancer. This meta-analysis suggests that miR-141 has a high diagnostic value for prostate cancer. In future, large-scale prospective studies will be done to verify and evaluate this result.
\end{abstract}

Keywords: miR-141, prostate cancer, meta-analysis

\section{INTRODUCTION}

Prostate cancer $(\mathrm{PCa})$ is one of the most common malignant tumors of the male urinary system. In $\mathrm{Eu}-$ ropean and American populations, its incidence rate ranks first and its death rate ranks second ${ }^{[1,2]}$. More than $50 \%$ of patients present at an advanced stage or have metastasis at diagnosis, which seriously affects their prognosis and quality of life ${ }^{[3]}$. In addition, due to poor sensitivity or specificity, it is difficult for currently available prostate-specific antigen(PSA) and other markers to meet clinical needs ${ }^{[4]}$. Therefore, identifying a new tumor marker with good sensitivity and specificity is important for the clinical diagnosis and treatment of $\mathrm{PCa}$.

miRNAs are involved in the regulation of many cellular processes, including the occurrence of tumors ${ }^{[5]}$. Some miRNAs can inhibit mRNA expression of recipient cells, leading to aberrant expression of target genes, thus transforming the microenvironment and promoting tumor development ${ }^{[6]}$. A large number of studies have confirmed the differential expression

*Correspondence to: Suliang Li, Department of Laboratory Medicine, the First Affiliated Hospital of Xi'an Medical University, Xi'an, Shaanxi, 710077, China. E-mail: lisuliang01@163.com.

Conflict of interests: The authors declare that there are no conflicts of competing interests. 
of miRNAs in tumor tissues, suggesting that miRNA expression profiles can be used as a biomarker for the early detection, classification and prognosis of tumors ${ }^{[7,8]}$. A previous study found that miRNA expression profiles were consistent with clinicopathological data and had better accuracy in distinguishing tumor tissues from normal tissues ${ }^{[9]}$. Moreover, miRNA has high stability, which makes it a good diagnostic tumor marker ${ }^{[10]}$. In recent years, miR-141 has gradually demonstrated its value in the diagnosis of prostate cancer and has become a potential new diagnostic marker ${ }^{[11]}$. However, the diagnostic parameters varied among previous studies ${ }^{[12]}$. Therefore, this systematic review was conducted to explore the diagnostic value of miR-141 in PCa.

\section{METHODS}

\section{Search strategy}

A meta-analysis was performed in accordance with Preferred Reporting Items for Systematic Reviews and Meta-Analyses (PRISMA) guideline ${ }^{[13]}$. A comprehensive search of literature in the PubMed, Medline, the Cochrane Library, and Embase databases up to October 31, 2019 was performed. The following search strategy was used: ("microRNA-141" or "miRNA-141" or "miR-141" or "CTC") and ("prostate cancer" or "prostate carcinoma" or "prostate neoplasm" or "prostate tumor"). Subsequently, eligible studies were included for further screening.

\section{Study selection}

Two researchers independently performed the literature search and study selection. Any disagreements were resolved by group discussion until a consensus was reached. Studies were included if they met the following study inclusion criteria: (1) miR-141 was involved in the study of patients who were diagnosed with $\mathrm{PCa}$; (2) patients with $\mathrm{PCa}$ were studied using the gold standard test (eg, a histological examination) for diagnosis; (3) serum, plasma or urine samples from PCa patients were used, and the control group involved patients with either benign prostate disease or healthy individuals; (4) sensitivity, specificity and critical values must be explicitly mentioned in the literature; and (5) the validation method and sufficient patients' information were reported. The exclusion criteria were as follows: (1) reviews, comments, letters and articles with an indefinite diagnostic threshold; (2) incomplete clinical data for extraction; (3) duplicate records; (4) experiments on DNA microarray; and (5) experiments on animals or cell lines.

\section{Data extraction}

The data was collected from the included studies and the following information was extracted: first author, publication year, country performed, age, sample number, sample type, detection method for miR141, area under receiver operating characteristic curve (AUC), and the sensitivity and specificity of miR-141 for diagnosing PCa.

\section{Quality assessment}

The Quality Assessment of Diagnostic Accuracy Studies (QUADAS-2) tool was used to evaluate the quality of studies included in the meta-analysis, which was performed independently by the two authors ${ }^{[14]}$. The method consisted of 4 components: patient selection, index test, reference standard, and flow and timing. The risk of bias for each item in individual studies can be judged as "low", "high" or "unclear", and is summarized by proportion. All authors agreed to the final determinants of the literature to be considered.

\section{Statistical analysis}

RevMan 5.3 was used to perform the quality assessment, and statistical software Stata version 12.0 (Stata Corp LP, College Station, TX, USA) and Meta-disc version 1.4 (version 1.4; Ramony Cajal Hospital, Madrid, Spain) were used to conduct other analyses. The sensitivity, specificity, positive likelihood ratio (PLR), negative likelihood ratio (NLR), diagnostic odds ratio (DOR) and corresponding $95 \%$ confidence intervals (CIs) were calculated from TP (true positive), FP (false positive), FN (false negative), and TN (true negative) cases, which were extracted from each study before data pooling. A bivariate random effects model ${ }^{[15]}$ was applied to summarize the sensitivity, specificity, PLR and NLR. A hierarchical regression model was used to summarize the summary receiver operating characteristic (ROC) curve and the area under the $\mathrm{ROC}^{[16]}$. The $Q$ statistic and $I^{2}$ was used to inspect the statistical heterogeneity across the eligible studies $\left(P\right.$-values $\leqslant 0.05$ and $I^{2}$-values $\geqslant 50 \%$ indicated heterogeneity for the $Q$ statistic $)^{[17]}$. Meta regression analyses were conducted on the basis of age, publication year, country, sample type and case number ${ }^{[18]}$. We used Deeks' asymmetry test to evaluate potential publication bias ${ }^{[19]}$ and Fagan's nomogram to evaluate the pretest probability and post-test probability of PLR and NLR ${ }^{[20]}$. All tests were two-sided, and a $P$-value $<0.05$ was considered statistically significant. 


\section{RESULTS}

\section{Literature search}

The results of the selection process are shown in Fig. 1. The initial search in the electronic databases yielded 116 relevant studies using the search strategy described above, of which 37 studies were excluded due to duplication. Additionally, 50 studies were excluded because they were letters, reviews, comments, animals or cell line studies, or had incomplete clinical data. After careful examination, 22 additional studies were excluded because they had index details missing or were not case-control studies. Finally, a total of 7 studies were included in the present meta-analysis.

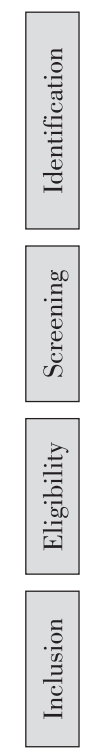

\section{Study characteristics}

The features of the enrolled studies are listed in Table 1. The seven studies were published between 2010 and 2018, including 279 PCa patients and 135 controls. Four studies were conducted in Europe ${ }^{[21-24]}$,

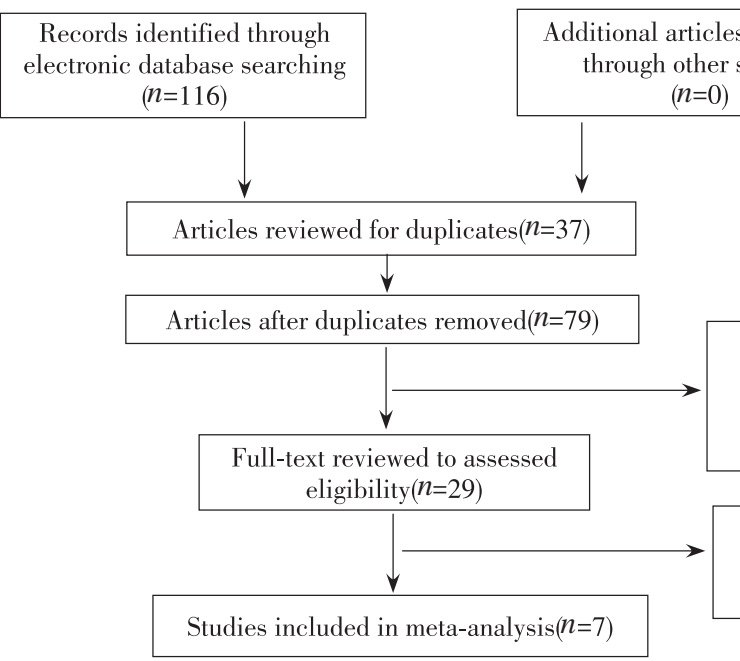

Studies were excluded because they were:

Letters,reviews, comments $(n=12)$

Experiments for animals and cell lines $(n=23)$

Incomplete clinical data $(n=15)$

Studies were excluded because they were:

Index deails missing $(n=14)$

Not case-control studies $(n=8)$

Fig. 1 Flow chart showing the study selection procedure.

Table 1 Characteristics of the eligible studies in the meta-analysis

\begin{tabular}{|c|c|c|c|c|c|c|c|c|c|}
\hline Author & Year & Country & Case(control), $n$ & Age, $y$ & Method & Sample & AUC & Sensitivity, $\%$ & Specificity, \% \\
\hline Pawel & 2018 & Poland & $20(8)$ & 68.6 & PCR & Serum & 0.831 & 65.0 & 88.0 \\
\hline Darina & 2015 & Bulgaria & $59(27)$ & 68 & PCR & Plasma & 0.567 & 56.5 & 57.1 \\
\hline Christa & 2014 & Denmark & $31(13)$ & - & PCR & Serum & 0.580 & 30.0 & 100.0 \\
\hline Brian & 2015 & Ireland & $75(27)$ & 64 & PCR & Plasma & 0.655 & 94.0 & 70.0 \\
\hline Zhuo & 2015 & China & $20(20)$ & 69.5 & PCR & Serum & 0.869 & 80.0 & 87.1 \\
\hline Nassin & 2018 & Iran & $23(20)$ & 68.4 & PCR & Urine & 0.850 & 88.0 & 93.0 \\
\hline
\end{tabular}

$\mathrm{PCR}=$ polymerase chain reaction, $\mathrm{AUC}=$ area under curve.

\section{Meta-analysis}

The summary results for sensitivity, specificity, PLR and NLR are presented in Fig. 3. The pooled sensitivity was 0.70 (95\% CI $0.64 \sim 0.75)$, the specifi city was 0.73 (95\% CI $0.64 \sim 0.80$ ), the PLR was 2.88 (95\% CI 1.40 5.93), and the NLR was 0.38 (95\% CI $0.20 \sim 0.71$ ). Further, the pooled DOR of miR-141 for prostate cancer was 9.94 (95\% CI: 2.55 38.80) (Fig. 4). Finally, the summary area under the ROC curve was 0.83 (95\% CI: 0.79 0.86) (Fig. 5).

\section{Heterogeneity analysis}

The pooled DOR was 9.94, with significant hetero- and three studies were conducted in Asia ${ }^{[25-27]}$. The quality assessment results of the included studies are shown in Fig. 2. The results showed that the included studies were of high quality and suitable for metaanalysis. 

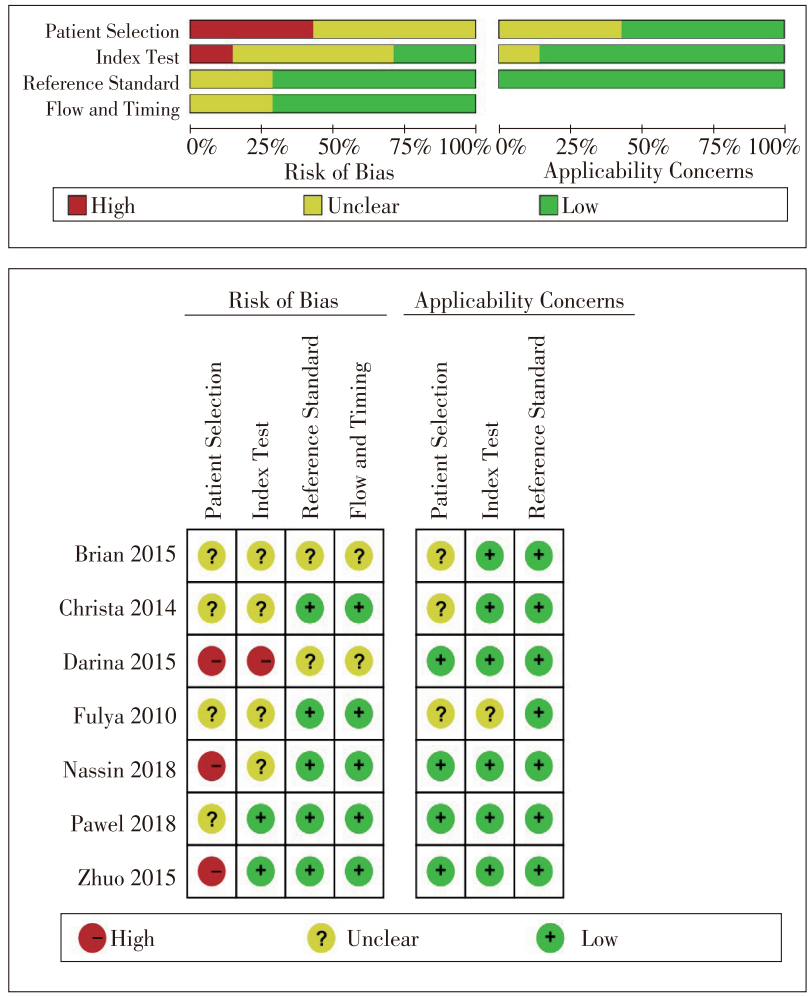

Fig. 2 Quality assessment of the included studies.
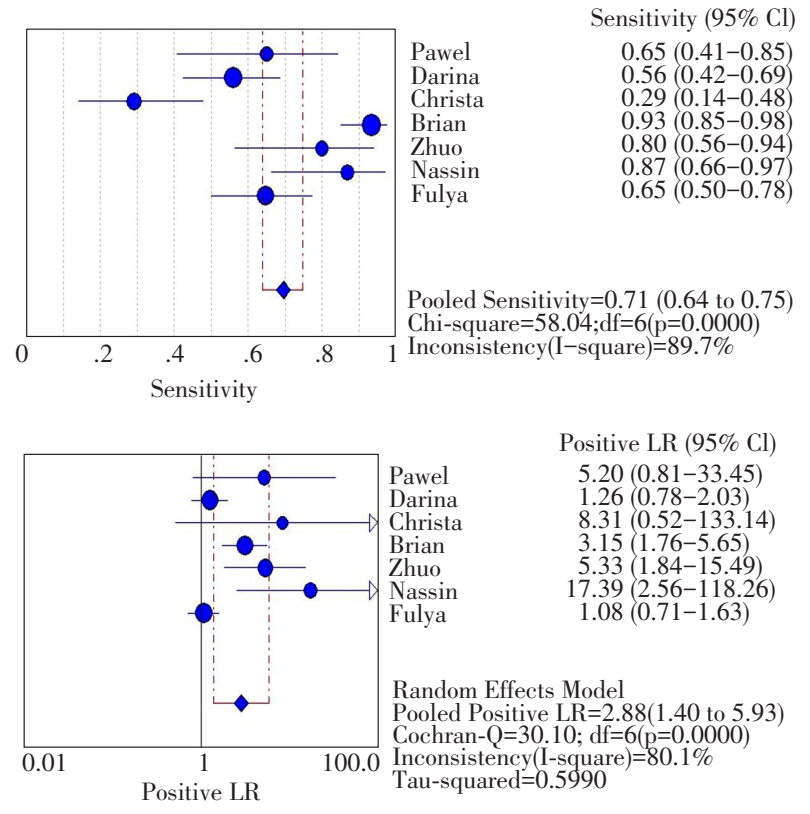

\section{Publication bias}

The Deeks' funnel plot asymmetry test was used to evaluate publication bias. The studies' funnel plots were symmetrical, and the results of the test showed no evidence of publication bias $(P=0.56$, Fig. 8 ).

\section{DISCUSSION}

In 2019, the estimated number of new cases of prostate cancer was 174,650 in the United States, accounting for $20 \%$ of all cases in men ${ }^{[28]}$. The early screening of prostate cancer can aid the early diagnosis and treatment of the disease, ultimately benefitting patients' prognosis ${ }^{[29]}$. The serum PSA test combined with digital rectal examination (DRE) is a widely recognized screening method for prostate cancer. Despite their widespread use, these tests have some limitations and drawbacks in the diagnosis of prostate cancer $^{[30]}$. Compared with the traditional detection method, microRNAs have shown better sensitivity and specificity in the diagnosis of prostate cancer ${ }^{[31]}$. In particular, miR-141 is considered to be the most effective biological marker for prostate cancer, wherein its expres-
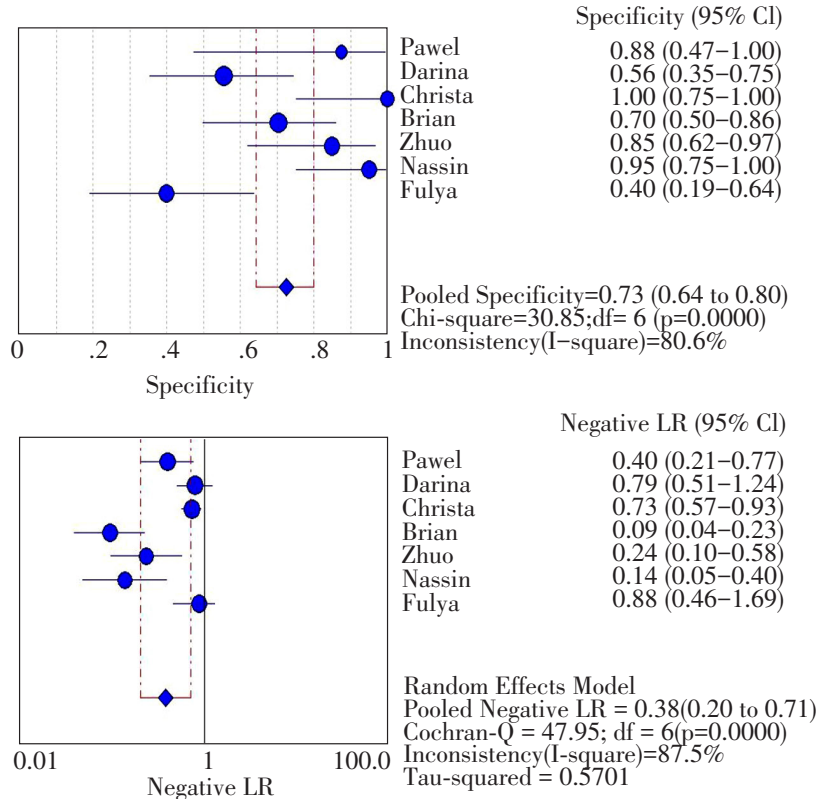

Fig. 3 Forest plots for sensitivity, specificity, PLR and NLR. PLR=positive likelihood ratio; NLR=negative likelihood ratio.

sion level was found to be 46 times higher than that of a normal control group ${ }^{[32]}$.

This meta-analysis evaluated the diagnostic value of miR-141 for prostate cancer and included 7 studies involving a total of 414 patients. The results showed that the pooled sensitivity and specificity were 0.70 and 0.73 , respectively, which showed that the diagnostic efficiency was high. In this study, the pooled PLR and NLR of miR-141 for prostate cancer were
2.88 and 0.38 , respectively, and the results showed an acceptable detection rate. These results suggest that the overall accuracy of prostate cancer detection by miR-141 is relatively good. Also, the pooled diagnostic odds ratio was 9.94, suggesting that miR141 has outstanding discrimination ability for prostate cancer. Furthermore, the area under the ROC curve was used to evaluate the overall diagnostic efficiency. The summary area under the ROC curve was 0.83 , 


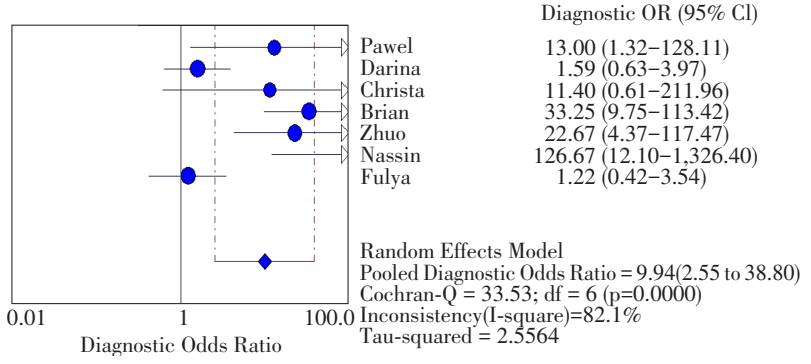

Fig. 4 Forest plot for DOR. DOR=diagnostic odds ratio.

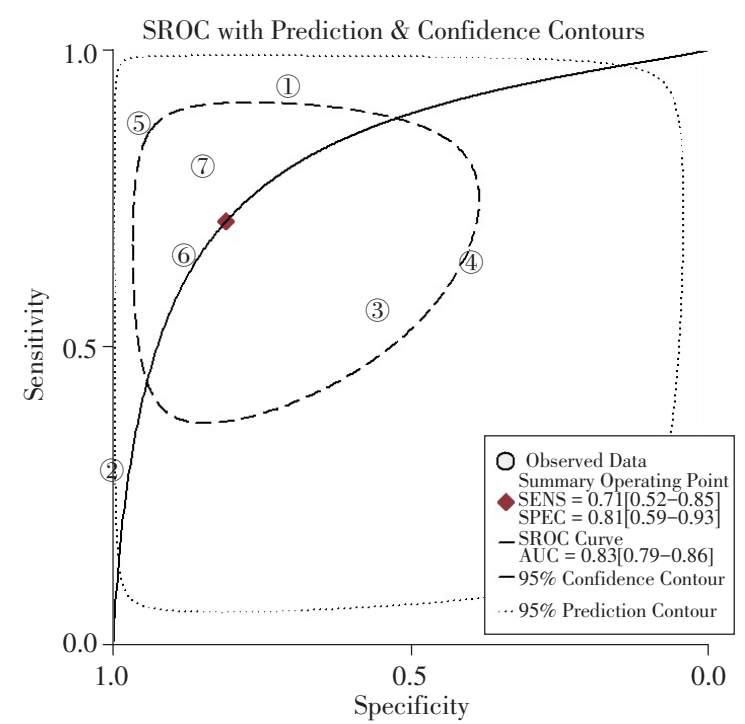

Fig. 5 sROC of miR-141 for the diagnosis of prostate cancer in all studies. $\mathrm{sROC}=$ summary receiver operating characteristic.

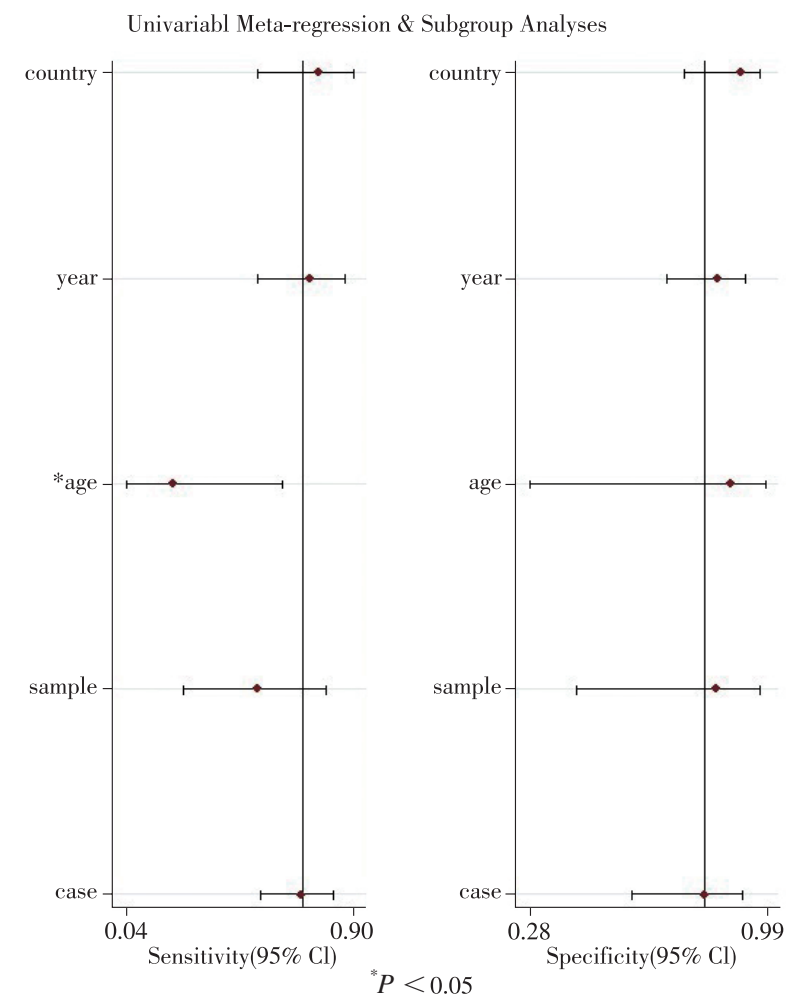

Fig. 6 Meta-regression analyses for DOR. DOR = diagnostic odds ratio.
Table 2 Meta-regression

\begin{tabular}{lrcccl}
\hline Variable & Coefficient & Std.Err. & $P$-value & RDOR & \multicolumn{1}{c}{$95 \%$ CI } \\
\hline Case & -0.03 & 0.03 & 0.25 & 0.97 & $0.90 \sim 1.04$ \\
Age & 1.94 & 1.41 & 0.01 & 6.96 & $0.14 \sim 344.15$ \\
Year & -1.98 & 1.56 & 0.27 & 0.14 & $0.00 \sim 10.54$ \\
Country & 0.52 & 0.12 & 0.24 & 1.68 & $1.21 \sim 2.34$ \\
Sample & -0.81 & 1.13 & 0.51 & 0.44 & $0.02 \sim 10.34$ \\
\hline
\end{tabular}

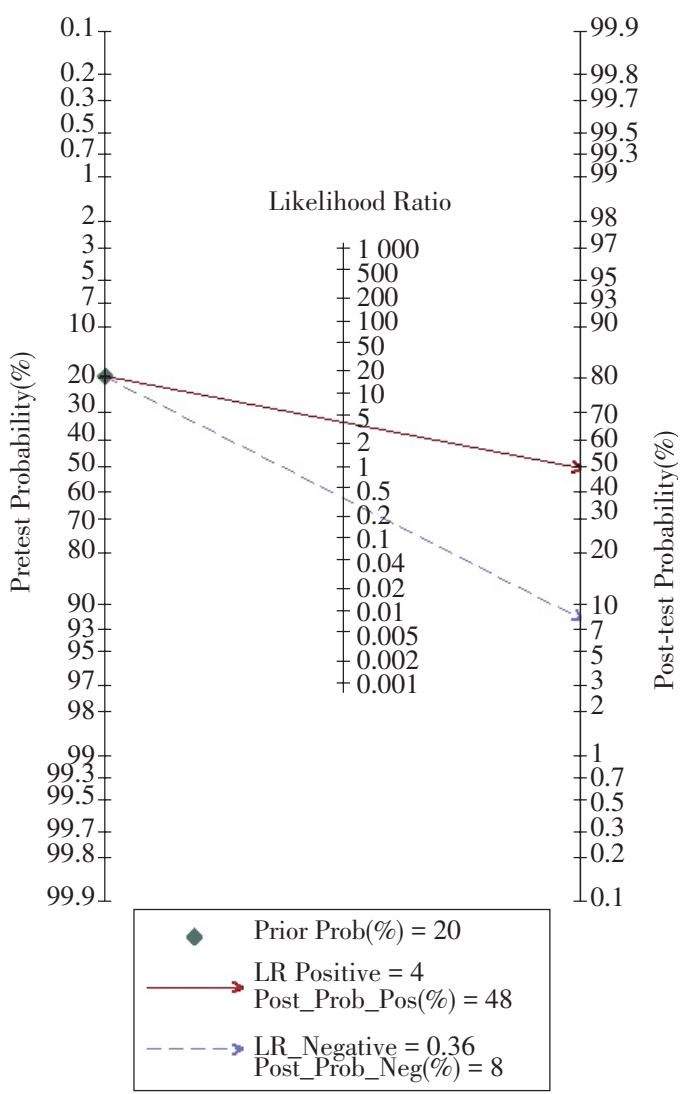

Fig. 7 Fagan's nomogram.

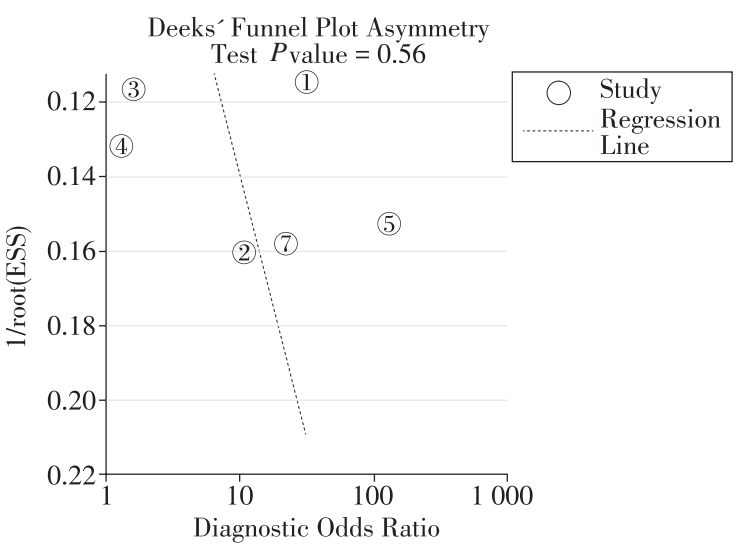

Fig. 8 Publication bias.

and the results indicated a high diagnostic value.

The $I^{2}$ value of the heterogeneity test of DOR was $82.1 \%$, which indicated high heterogeneity. Therefore, meta-regression analysis was used to explore the possible sources of heterogeneity. The $P$-value of age was 0.01 , and the remaining $P$-values were all $>0.05$. This suggested that the patients' age was the main source 
of heterogeneity. The Deeks' funnel plot asymmetry test showed no evidence of publication bias $(P=0.56)$.

The Fagan nomogram results showed a significant increase when using miR-141 for diagnosing prostate cancer. Upregulated miR-141 is suspicious for lung cancer, increasing the pretest probability of the PLR from $20 \%$ to a post-test probability of $48 \%$. Furthermore, upregulated miR-141 decreased the pretest probability from $20 \%$ to $8 \%$. Since miRNAs were first identified in 1993, many studies have examined whether the expression of certain miRNAs in prostate cancer is associated with tumor risk and progre $\operatorname{ssion}^{[33,34]}$. Based on the results of the current metaanalysis, we believe that the detection of miR-141 combined with conventional methods can help doctors treat and monitor disease progression. The detection of miR-141 is helpful for the diagnosis and treatment of prostate cancer. Studies of miRNAs are however, still at the design stage and there are still many cha llenges in its clinical application. The next step will undoubtedly need to involve a large number of studies, in order to further improve the detection sensiti vity and specificity of miR-141, and provide a wider scientific basis for clinical practice.

This meta-analysis study suggests that miR-141 has a high diagnostic value for prostate cancer. In future, large-scale prospective studies will be done to verify and evaluate this result.

\section{Acknowledgments}

This study was supported by the grant of Education Department of Shaanxi Provincial Government (20JK0723). The authors express their gratitude to the study participants and research personnel for their involvement in the study.

\section{References}

[1] Ferlay J, Shin HR, Bray F, et al. Estimates of worldwide burden of cancer in 2008: GLOBOCAN 2008[J]. Int $J$ Cancer, 2010, 127(12): 2893-917.

[2] Siegel RL, Miller KD, Jemal A. Cancer statistics, 2015[J]. CA Cancer J Clin, 2015, 65(1): 5-29.

[3] Wang Y, Guo J, Xu L, et al. Should bone scan be performed in Chinese prostate cancer patients at the time of diagnosis?[J]. Urol Int, 2013, 91(2): 160-4.

[4] Obort AS, Ajadi MB, Akinloye O. Prostate-specific antigen: any successor in sight? [J]. Rev Urol, 2013, 15(3): 97-107.

[5] Krol J, Loedige I, Filipowicz W. The widespread regulation of microRNA biogenesis, function and decay [J]. Nat Rev Genet, 2010, 11(9): 597-610.

[6] Song CJ, Chen H, Chen LZ, et al. The potential of microRNAs as human prostate cancer biomarkers: a metaanalysis of related studies[J]. J Cell Biochem, 2018,
119(3): 2763-86.

[7] Chen M, Calin GA, Meng QH. Circulating microRNAs as promising tumor biomarkers[J]. Adv Clin Chem, 2014, 67: 189-214.

[8] Peng J, Xie Z, Cheng L, et al. Paired design study by realtime PCR: miR-378* and miR-145 are potent early diagnostic biomarkers of human colorectal cancer[J]. BMC Cancer, 2015, 15: 158.

[9] Lu J, Getz G, Miska EA, et al. MicroRNA expression profiles classify human cancers[J]. Nature, 2005, 435(7043): 834-8.

[10] Mishra S, Deng JJ, Gowda PS, et al. Androgen receptor and microRNA-21 axis downregulates transforming growth factor beta receptor II (TGFBR2) expression in prostate cancer[J]. Oncogene, 2014, 33(31): 4097-106.

[11] Ghorbanmehr N, Gharbi S, Korsching E, et al. miR-21$5 \mathrm{p}, \mathrm{miR}-141-3 \mathrm{p}$, and $\mathrm{miR}-205-5 \mathrm{p}$ levels in urinepromising biomarkers for the identification of prostate and bladder cancer[J]. Prostate, 2019, 79(1): 88-95.

[12] Song CJ, Chen H, Chen LZ, et al. The potential of microRNAs as human prostate cancer biomarkers: a metaanalysis of related studies[J]. J Cell Biochem, 2018, 119(3): 2763-86.

[13] Moher D, Liberati A, Tetzlaff J, et al. Preferred reporting items for systematic reviews and meta-analyses: The PRISMA statement[J]. PLoS Med, 2009, 6: e1000097.

[14] Whiting PF, Weswood ME, Rutjes AW, et al. Evaluation of QUADAS, a tool for the quality assessment of diagnostic accuracy studies[J]. BMC Med Res Methodol, 2006, 6: 9.

[15] DerSimonian R, Laird N. Meta-analysis in clinical trials[J]. Control Clin Trials, 1986, 7(3): 177-88.

[16] Walter SD. Properties of the summary receiver operating characteristic (SROC) curve for diagnostic test data[J]. Stat Med, 2002, 21(9): 1237-56.

[17] Jackson D, White IR, Riley RD. Quantifying the impact of between-study heterogeneity in multivariate metaanalyses[J]. Stat Med, 2012, 31(29): 3805-20.

[18] Altman DG, Bland JM. Interaction revisited: the difference between two estimates[J]. BMJ, 2003, 326: 219.

[19] Deeks JJ, Macaskill P, Irwig L. The performance of tests of publication bias and other sample size effects in systematic reviews of diagnostic test accuracy was assessed[J]. J Clin Epidemiol, 2005, 58: 882.

[20] Akobeng AK. Understanding diagnostic tests 2: likelihood ratios, pre- and post-test probabilities and their use in clinical practice[J]. Acta Paediatr, 2007, 96(4): 487-91.

[21] Porzycki P, Ciszkowicz E, Semik M, et al. Combination of three miRNA (miR-141, miR-21, and miR$375)$ as potential diagnostic tool for prostate cancer recognition[J]. Int Urol Nephrol, 2018, 50(9): 1619-26.

[22] Kachakova D, Mitkova A, Popov E, et al. Combinations of serum prostate-specific antigen and plasma expression levels of let-7c, miR-30c, miR-141, and miR375 as potential better diagnostic biomarkers for prostate cancer[J]. DNA Cell Biol, 2015, 34(3): 189-200.

[23] Haldrup C, Kosaka N, Ochiya T, et al. Profiling of circulating microRNAs for prostate cancer biomarker 
discovery[J]. Drug Deliv Transl Res, 2014, 4(1): 19-30.

[24] Kelly BD, Miller N, Sweeney KJ, et al. A circulating microRNA signature as a biomarker for prostate cancer in a high risk group[J]. J Clin Med, 2015, 4(7): 1369-79.

[25] Li Z, Ma YY, Wang J, et al. Exosomal microRNA-141 is upregulated in the serum of prostate cancer patients[J]. Onco Targets Ther, 2015, 9: 139-48.

[26] Ghorbanmehr N, Gharbi S, Korsching E, et al. miR-21$5 \mathrm{p}, \mathrm{miR}-141-3 \mathrm{p}$, and miR-205-5p levels in urinepromising biomarkers for the identification of prostate and bladder cancer[J]. Prostate, 2019, 79(1): 88-95.

[27] Yaman Agaoglu F, Kovancilar M, Dizdar Y, et al. Investigation of miR-21, miR-141, and miR-221 in blood circulation of patients with prostate cancer[J]. Tumour Biol, 2011, 32(3): 583-8.

[28] Siegel RL, Miller KD, Jemal A. Cancer statistics, 2019[J]. CA Cancer J Clin, 2019, 69(1): 7-34.

[29] Lima AR, Pinto J, Azevedo AI, et al. Identification of a biomarker panel for improvement of prostate cancer diagnosis by volatile metabolic profiling of urine[J]. $\mathrm{Br} \mathrm{J}$ Cancer, 2019, doi: 10.1038/s41416-019-0585-4.
[30] Szeliski K, Adamowicz J, Gastecka A, et al. Modern urology perspectives on prostate cancer biomarkers[J]. Cent European J Urol, 2018, 71(4): 420-6.

[31] Yin C, Fang C, Weng H, et al. Circulating microRNAs as novel biomarkers in the diagnosis of prostate cancer: a systematic review and meta-analysis[J]. Int Urol Nephrol, 2016, 48(7): 1087-95.

[32] Mitchell PS, Parkin RK, Kroh EM, et al. Circulating microRNAs as stable blood-based markers for cancer detection[J]. Proc Natl Acad Sci USA, 2008, 105(30): 10513-8.

[33] Shi XB, Xue L, Yang J, et al. An androgen-regulated miRNA suppresses Bakl expression and induces androgen-independent growth of prostate cancer cells[J]. Proc Natl Acad Sci USA, 2007, 104(50): 19983-8.

[34] Li SL, Ye Y, Wang SY, et al. Exosomal miR-375 promotes the activity of osteoblasts in prostate cancer[J]. Asia-Pacific Journal of Blood Types and Genes, 2018, 2(4): 241-8.

Received 9 December 2019, Revised 7 January 2020, Accepted 31 January 2020 